\title{
POLÍTICAS PÚBLICAS PARA EL CONTROL DE ENFERMEDADES TRANSMITIDAS POR VECTORES EN MÉXICO.
}

\author{
Juache Villagrana Alan Esteban*, Flores Suárez Adriana E.*
}

*Universidad Autónoma de Nuevo León, Facultad de Ciencias Biológicas. México.

Citation: Juache Villagrana A.E., Flores Suárez A.E. (2021) Políticas públicas para el control de enfermedades transmitidas por vectores en México. Revista Salud Pública y Nutrición, 20 (2), 42-51.

Editor: Esteban G. Ramos Peña, Dr. CS., Universidad Autónoma de Nuevo León, Facultad de Salud Pública y Nutrición, Monterrey Nuevo León, México. Copyright: @2021 Juache Villagrana A.E., et al. This is an open-access article distributed under the terms of Creative Commons Attribution License [CC BY 4.0], which permits unrestricted use, distribution, and reproduction in any medium, provided the original author and source are credited.

Competing interests: The authors have declared that no competing interests exist.

DOI: https://doi.org/10.29105/respyn20.2-5

Recibido: 04 de diciembre 2020; Aceptado: 19 de febrero 2021

Email: adriana.floressr@uanl.edu.mx 


\title{
POLÍTICAS PÚBLICAS PARA EL CONTROL DE ENFERMEDADES TRANSMITIDAS POR VECTORES EN MÉXICO.
}

\author{
Juache Villagrana Alan Esteban *, Flores Suárez Adriana E. * \\ *Universidad Autónoma de Nuevo León, Facultad de Ciencias Biológicas. México.
}

\section{RESUMEN}

Introducción. Las enfermedades transmitidas por vectores (ETV) persisten como un problema nacional de salud pública. A fin de disminuir su impacto, existen políticas públicas dirigidas al manejo de estas infecciones. Las soluciones propuestas, así como su implementación, se estipulan dentro de documentos como la ley general de salud (LGS) y normas oficiales mexicanas (NOM). Pese a que la ejecución de las políticas públicas ha conducido a resultados favorables en contra de las ETV, existen nuevas pautas internacionales establecidas por la Organización Mundial de la Salud (OMS) que incrementarían la eficacia de las políticas actuales. Objetivo: Contrastar las acciones estipuladas en las políticas públicas mexicanas del 2000 a 2020 para el combate a las ETV contra las directrices internacionales actuales. Conclusiones: La mayoría de las actividades en contra de las ETV recaen dentro del control de vectores, mismo que al ser comparado con las directrices de la OMS exhibe áreas de oportunidad en la escala de aplicación, uso de insecticidas, participación comunitaria, investigación, entre otras. Bajo este escenario, las políticas públicas deben actualizarse para incluir nueva información propuesta por organismos internacionales. Palabras Clave: Control de vectores, manejo de vectores, legislación mexicana.

\section{ABSTRACT}

Introduction: Vector-borne diseases (VBD) persist as a national public health problem in many countries including Mexico. To avoid their impact in public health there is a series of public policies oriented directly or indirectly towards their control. All proposed solutions and their implementation are written in official documents such as the Mexican general law of health and official norms. Even though enforcement of public policies has brought positive results versus VBD, there are new guidelines elaborated by the World Health Organization (WHO) that can boost actual efforts. Objective: To contrast actions provided by Mexican public policies of 2000-2020 regarding combat of VBD with those guidelines supplied by the WHO. Conclusions: Most actions taken against VBD are vector control methods using integrated vector management as a guide methodology. However, a comparison of actual integrated vector management implemented in Mexico to global alignments proposed by the WHO exhibit improvements that must be applied to enhance control of VBD. Some of these advancements include the scale of application of public policies, insecticide use and management, community participation, VBD research, and others. In this framework, public policies should be updated to include new information suggested by international entities. Key words: Vector control, vector management, Mexican legislation. 


\section{Introducción}

En diversas partes del mundo, incluyendo México, las enfermedades transmitidas por vectores persisten como un problema de salud pública. Su impacto es evidente tanto por la cantidad de personas afectadas, así como por las consecuencias económicas que generan. Por ejemplo, en 2018 se registraron 228 millones de casos (206-258 millones, IC $95 \%$ ) de paludismo, de los cuales ocurrieron un total de 405 000 consecuencias fatales a nivel mundial (OMS, 2019). Siguiendo con este ejemplo, el impacto económico se evidencia en los países con mayor prevalencia quienes tienen un crecimiento económico menor en comparación a países libres de paludismo (Gallup y Sachs, 2001). Debido a las consecuencias negativas de las ETV existen organismos gubernamentales y no gubernamentales encargados de aminorar y prevenir tales consecuencias por medio del desarrollo de políticas públicas. Desde esta perspectiva, las políticas públicas, tal y como lo define Velásquez (2009), son procesos que incluyen las decisiones, acciones, inacciones, acuerdos e instrumentos, que buscan solucionar o prevenir una situación identificada como problemática. En México, todas las estrategias propuestas para el combate a las ETV están establecidas dentro de documentos normativos oficiales tales como la ley general de salud y diversas normas oficiales. En este mismo contexto, las acciones planteadas para el manejo de ETV datan de siglos pasados, no obstante, pese a que la ejecución de las políticas públicas ha conducido a resultados favorables en contra de las ETV, existen nuevas pautas internacionales establecidas por la Organización Mundial de la Salud (OMS) que incrementarían la eficacia de las políticas actuales. En este ensayo se describe el panorama en el que se encuentran las ETV en territorio nacional dentro del periodo de 2000 al 2020, además, se enuncia el fundamento legal oficial para el manejo de estas infecciones dictado en la constitución política, la ley general de salud, y normas oficiales mexicanas establecidas dentro del mismo periodo de tiempo. Por último, ya que los esfuerzos mayoritarios para el control de las ETV se dan en el control de vectores, se contrastan las acciones específicas de las políticas públicas con los lineamientos internacionales del manejo integrado de vectores.

\section{Panorama de las ETV en México}

Previo a la descripción de las ETV como problema de salud pública en México, es importante describir rasgos básicos de este grupo de enfermedades. La característica distintiva de las ETV es la transmisión de patógenos mediada por dípteros (mosquitos y moscas), ftirapteros (piojos), hemipteros (chinches), e ixodidos (garrapatas) (Organización Mundial de la Salud [OMS], 2020). Estos artrópodos, conocidos como vectores, actúan como vehículos de agentes infecciosos que transmiten a huéspedes vertebrados durante interacciones como la alimentación hematófaga (Mullen y Durden 2009). Los virus de la fiebre amarilla y dengue, diseminados por dipteros del género Aedes (Patterson et al., 2016), o bien, el parásito Trypanosoma cruzi agente causal de la enfermedad de Chagas transmitido por chinches de los géneros Triatoma y Rhodnius, son ejemplos de estos patógenos (González y Hernández, 2017; Pita et al., 2018). Establecida la relación tripartita entre patógeno-vector-huésped, para reducir el impacto de las ETV existen técnicas de control, como vacunas implementadas en contra del dengue (Sridhar et al., 2018), o métodos quimioprofilácticos en el caso del paludismo (Schwartz, 2012). No obstante, el control de poblaciones de vectores permanece como el método de primera elección para el manejo de las ETV. Cada país está encargado de aplicar las estrategias de control que considere pertinentes de acuerdo con sus políticas públicas, en función de las situaciones epidemiológicas y entomológicas de cada región. Ahora bien, en México diversas ETV han sido reconocidas como problema de salud pública a través del tiempo. Los casos nuevos de estas enfermedades son registrados dentro del boletín epidemiológico (BE) del Sistema Nacional de Vigilancia Epidemiológica (SINAVE). En el periodo 2000-2002 se reportaba la morbilidad del paludismo (provocado por Plasmodium falciparum y P. vivax), el dengue y dengue hemorrágico. A partir de 2003 y hasta 2013 se reportaba la morbilidad de estas infecciones en conjunto con los casos confirmados de fiebre del Oeste del Nilo. En 2014 se incluyeron los casos confirmados de tifo epidémico, tifo murino, otras rickettsiosis, e infecciones de fiebre manchada. En 2015 y hasta 2016 fue incorporada la enfermedad por virus del Zika. A partir del 2017 a la fecha, los boletines epidemiológicos contienen las ETV mencionadas anteriormente y los casos de enfermedad por virus chikungunya. A la par, otras ETV son clasificadas como de interés local, regional 
o institucional que no son reportadas dentro del BE. Es relevante mencionar que la vigilancia de la mayoría de estas ETV ocurre de manera obligatoria de acuerdo con normativas oficiales mexicanas tales como la NOM-017-SSA2-2012 (Diario Oficial de la Federación [DOF], 2013). Los reportes oficiales de cifras de nuevos casos emitidos en el boletín epidemiológico nacional muestran que existe una variación considerable en cuanto a la persistencia de las ETV dentro del territorio mexicano; en https://alanjuache.wixsite.com/etvmexico se puede visualizar en número de casos confirmados de las ETV vigiladas en México y su variación temporal.

\section{Sustento oficial para la vigilancia y control de las} ETV en México

En México existe una legislación orientada al problema que representan las ETV, primordialmente el artículo $4^{\circ}$ de la Constitución Política de los Estados Unidos Mexicanos (DOF, 2020) establece la protección de la salud como derecho personal. Este artículo da paso al surgimiento de la Ley General de Salud (LGS, 2020), en donde se detalla el acceso a los servicios de salud y las condiciones de salubridad general. Esta ley contiene artículos relacionados directamente a las ETV. De inicio, el artículo $2^{\circ}$ instituye el derecho a la protección de la salud y en su fracción VIII, dicta la prevención de las enfermedades para todas las personas. En materia de Salubridad General, la fracción XV del artículo $3^{\circ}$ señala la prevención y el control de las enfermedades transmisibles donde se incluye a las ETV. En el artículo $28^{\circ}$ se menciona el Compendio Nacional de Insumos para la Salud, mismo que es aprobado por la Secretaría de Salud (SS), el cual contiene la lista de insumos esenciales para la protección de los mexicanos. En este compendio se incluyen los plaguicidas aplicados para el control de vectores; aprobados por un órgano de la SS, el Centro Nacional de Programas Preventivos y Control de Enfermedades (CENAPRECE). De manera similar, dentro de la La Ley General de Salud (LGS) se establece que la SS es encargada de establecer y operar el Sistema Nacional de Vigilancia Epidemiológica (artículo 133, fracción II).

El artículo 134 acuerda que tanto la SS, así como los gobiernos estatales, deben realizar actividades de vigilancia epidemiológica, prevención y control de las siguientes ETV: fiebre amarilla, dengue y otras arbovirosis (fracción VI), paludismo, tifo, fiebre recurrente transmitida por piojo, otras rickettsiosis, leishmaniasis, tripanosomiasis y oncocercosis (fracción VII). Algunas de estas infecciones tienen requisitos especiales sujetos al Reglamento Sanitario Internacional (OMS, 2008) y a un reglamento interno de la LGS en materia de Sanidad Internacional (DOF, 1985), de manera que, infecciones individuales de fiebre amarilla deben reportarse a la SS o la autoridad sanitaria más cercana de manera inmediata (artículo 136, fracción I), al igual que cualquier brote o epidemia de cualquier enfermedad (fracción II). Siguiendo estos reglamentos, otras enfermedades deben notificarse en un plazo no mayor a $24 \mathrm{~h}$, incluyendo los casos individuales de tifo epidémico, fiebre recurrente transmitida por piojo, paludismo, y casos humanos de encefalitis equina venezolana (fracción III). Por último, cualquier infección debe ser notificada en un plazo no mayor a $24 \mathrm{~h}$ ubicando los primeros casos individuales de otras enfermedades transmisibles en un área no infectada (fracción IV). La LGS obliga a la confirmación por cualquier medio clínico disponible cualquier enfermedad diagnosticada (artículo 139, fracción I).

La primera acción concreta para el control de ETV se da en la LGS, que establece como medida preventiva, la destrucción o control de vectores, reservorios y fuentes de infección naturales o artificiales que representen un riesgo para la salud (artículo 139, fracción VI). Este control de vectores ocurre por diferentes técnicas, como la aplicación de insecticidas. En México, la LGS ejerce una regulación sobre los plaguicidas, sustancias o mezclas de sustancias que permiten controlar organismos perjudiciales que comprenden, desde plagas agrícolas hasta vectores de enfermedades humanas o animales (artículo 278, fracción I). En cuanto a los plaguicidas útiles, la LGS refiere en su artículo 279 que la SS es la encargada de autorizar los productos que pueden contener plaguicidas, sus disolventes, procesos de persistencia y bioacumulación (fracción II-IV) y finalmente, las NOM que contemplen lo referente a la fabricación, manejo, almacenamiento, comercialización, y aplicación (fracción V). Para concluir, el artículo 404 se decreta la destrucción o el control de insectos u otra fauna transmisora y nociva (fracción VI).

Normas Oficiales Mexicanas enfocadas a las ETV La primera norma enfocada a las enfermedades transmisibles es la NOM-017-SSA2-2012 "Para la 
Vigilancia Epidemiológica" (DOF, 2013). En esta norma se especifican los criterios, designaciones y directrices operacionales del Sistema Nacional de Vigilancia Epidemiológica. Aquí se establece que es obligatorio vigilar la morbilidad de: la encefalitis equina venezolana, fiebre amarilla, fiebre hemorrágica por dengue, fiebre por dengue, fiebre manchada, fiebre del oeste del Nilo, peste, el paludismo por $\mathrm{P}$. falciparum y $\mathrm{P}$. vivax, tifo epidémico y murino, y otras rickettsiosis. Todas estas ETV se notifican de manera semanal, sin embargo, la encefalitis equina venezolana, fiebre amarilla, fiebre hemorrágica por dengue, fiebre manchada, fiebre por oeste del Nilo, peste, el paludismo por P. falciparum, y los tifos, presentan además notificación inmediata. Todas las ETV mencionadas tienen vigilancia de mortalidad, mientras que solo algunas como la fiebre hemorrágica y no hemorrágica por dengue y el paludismo presentan vigilancia especial. En este documento se estipulan las metodologías de vigilancia como la forma convencional, la basada en laboratorio, centinela, entre otras. Otras ETV clasificadas como de interés local, regional o institucional son la leishmaniasis visceral y cutánea, oncocercosis y la enfermedad por virus del chikungunya.

La NOM-032-SSA2 (DOF, 2015) es el documento normativo que dicta las acciones definitivas para el control de ETV. Desde su primera expedición en 2002 ha tenido dos modificaciones, una en 2010 y otra en 2014, siendo esta ultima la vigente. Su objetivo principal es delimitar criterios y procedimientos para disminuir riesgo de infecciones y las complicaciones de las ETV. En la primera parte de esta norma se promueve la concientización poblacional sobre las características sociales de las ETV y cómo estas influyen en la transmisión y prevención de enfermedades. De igual forma, presenta una descripción del manejo integrado de vectores (MIV), estrategia que definen como el uso de dos o más metodologías de control de vectores priorizando el uso de aquellas con menor impacto ambiental negativo y dejando como último recurso los agentes químicos sintéticos. Dichas metodologías engloban el control físico, químico, biológico, botánico, misceláneo y regulatorio. El control químico por medio de compuestos sintéticos utiliza como base la lista de productos recomendados autorizada por el CENAPRECE. Esta lista es actualizada con base en la eficacia de los productos analizada a través de monitoreos de susceptibilidad y pruebas de efectividad biológica. Los monitoreos de efectividad corresponden al esquema de evaluación de plaguicidas (WHOPES, por sus siglas en ingles) de la OMS y generalmente solo se utilizan mosquitos Ae. aegypti como organismos de referencia. Adicionalmente, esta NOM ordena la vigilancia entomológica a fin de estimar la densidad o abundancia de vectores.

Esta norma presenta apartados especiales para las ETV de mayor importancia en el país. Para el MIV de dengue, se recomienda el uso de medios físicos, químicos y biológicos para reducir las poblaciones de vectores. Particularmente, el control físico se implementa con barreras físicas para evitar el ingreso de mosquitos a casas, la limpieza, y que el agua almacenada esté libre de etapas inmaduras de mosquitos. En cuanto al uso de insecticidas se recomienda la combinación de larvicidas y adulticidas de cualquier tipo. En el caso del paludismo se recomiendan los mismos procedimientos de vivienda segura. La aplicación de insecticidas se efectúa solo en caso de brotes o después de desastres naturales utilizando larvicidas y adulticidas. La aplicación se realiza principalmente focalizada a casas continuamente positivas a paludismo (casas palúdicas). La enfermedad de Chagas tiene un MIV encaminado al control de la vivienda mediante su mejoramiento, medidas de manipulación, o modificación permanente. El control químico se realiza por cualquier insecticida que demuestre efectividad biológica de aplicación residual en muros internos y externos, así como estructuras peridomiciliares justo antes de la época de lluvias según el nivel de infestación. La oncocercosis tiene un control por medio de la administración de ivermectina en dosis semestrales; a partir de 2015 es catalogada como ETV eliminada en el territorio nacional. Para sitios donde se han detectado casos de leishmaniasis cutánea localizada, mucocutánea y difusa se recomienda el uso de ropa para evitar la exposición de la piel, medida complementada con el uso de repelentes. Solo para casos reportados de leishmaniasis visceral se recomienda el uso de insecticidas residuales aplicados en las viviendas, al igual que el uso de pabellones en la población en riesgo. Los mosquitos vectores de virus del oeste del Nilo son controlados por medio de insecticidas de acción efímera, larvicidas y medidas de control físico sobre los 
criaderos. Por último, el manejo integrado de vectores de Rickettsia incluye una combinación de estrategias de vivienda segura, ectodesparasitantes en fauna nociva como perros callejeros o propios, además del control químico por medio de rociado espacial con plaguicidas efímeros en sitios con casos probables.

\section{Lineamientos mundiales sobre MIV y su contraste con México}

El MIV es un concepto utilizado desde hace tiempo, sus principios fundamentales tienen origen en diferentes partes del mundo a partir del control de mosquitos. Beier et al. (2008) señalan los siete elementos principales del MIV: la reducción de vectores y transmisión de patógenos, actividades de control seguras a nivel ecológico, ambiental, social, económico y político, búsqueda de tácticas de control sin efectos negativos, desarrollo de resistencia, y la afectación a solo organismos objetivo, conocimiento del ciclo de transmisión de las ETV y biología de vectores, programas efectivos con métodos predictivos para la dinámica poblacional y de transmisión, control dinámico aplicando diversas estrategias, y por último, el dinamismo de las estrategias debe darse acorde a un programa de vigilancia de vectores y patógenos activo. A fin de facilitar la adopción del MIV para el control de ETV en distintas regiones del mundo, la OMS ofrece un manual-guía de esta metodología (OMS, 2012), mismo que será utilizado como base para comparar la legislación en México y su adecuación posterior para cumplir los retos de la respuesta mundial 20172030. Además de estas directrices mundiales, la Organización Panamericana de la Salud (OPS) presenta una guía operativa sobre la aplicación del MIV para las Américas (OPS, 2019) la cual servirá de punto de referencia a nivel regional.

A continuación, presentamos una breve reseña de este manual resaltando aquellos conceptos clave de planeación, implementación y vigilancia aplicados para un correcto MIV. Consideramos que varios de estos conceptos no han sido atendidos a cabalidad por las autoridades sanitarias mexicanas, condiciones que discutimos más adelante. Los principales puntos a tomar en cuenta para la adopción del MIV como estrategia en contra de las ETV incluyen: la toma de decisiones basada en evidencias, la atención de las ETV de manera integral y no individualista, el ajuste de las medidas hacia el cambio climático y la urbanización, la concientización de los agricultores y la comunidad en general sobre su impacto en las ETV y la existencia de una limitada cantidad de insecticidas para control de vectores que puede desencadenar la subsecuente aparición de resistencia. Utilizando estos parámetros el MIV es una aproximación que tiende a resolver problemas empleando información obtenida de campo y de la cual la resolución espacial es importante; entre más local sea el área de recolección de datos, las intervenciones serán más efectivas. La implementación de un MIV efectivo, por consiguiente, debe cumplirse en un eje que incluya la participación social y legislativa, la cooperación intra e intersectorial, una orientación integral para eficientizar los recursos. Su aplicación debe ocurrir en una escala local con coordinación flexible a niveles municipales, estatales y federal que asegure la mejor toma de decisiones basadas en las condiciones entomológicas y epidemiológicas focalizadas.

Partiendo desde la planeación del MIV una de las principales bases a considerar son las políticas públicas en contra de las ETV. Las políticas públicas permiten un accionar correcto y deben contemplar la política de salud nacional, integración de los sistemas de salud, guías de control de vectores, y el uso de plaguicidas. Las políticas públicas mexicanas incluyen la LGS, las normas y otros documentos como los lineamientos operativos de vigilancia de ETV. Dentro de esta normatividad debe existir una flexibilidad legislativa para que se considere a nivel federal como la parte administrativa que establece las políticas y guías de acción, pero que la elección e implementación de las medidas sea dictada por el nivel municipal o su escalón más próximo. Si bien en la LGS se estipula que como autoridades sanitarias se encuentran el presidente de la Republica, el consejo de salubridad general, la secretaria de salud y los gobiernos de las entidades federativas (artículo 4 , todas las fracciones), no se describen a mayor detalle el límite de las atribuciones de cada uno de ellos. Lo anterior supone un problema de flexibilidad ya que todo es controlado a nivel estatal o federal desestimando el límite de autoridad del nivel local. Esto puede verse reflejado en otros aspectos del MIV como el uso de insecticidas. Las pautas para el uso y la aplicación de plaguicidas dependen del nivel federal vía el CENAPRECE y abarca desde su autorización por medio de la lista de insumos 
recomendados hasta los monitoreos de resistencia. Históricamente no se realizan estudios activos sobre monitoreo de resistencia en otras especies distintas a Ae. aegypti. Asimismo, todas las pruebas de efectividad biológica se realizan en esta especie dejando un vacío de información en la funcionalidad de los plaguicidas sobre otros vectores como las chinches transmisoras del mal de Chagas. En conjunto, los problemas de inflexibilidad de políticas públicas en una escala local y el ineficiente manejo de plaguicidas ocasionan un mayor problema. Siguiendo con el ejemplo de la enfermedad de Chagas, se estima que esta ETV afecta a 1.1 millones de personas en México en donde la transmisión no ocurre de manera homogénea en toda la república siendo los estados con mayor incidencia Yucatán, Oaxaca e Hidalgo (Rojo, et al. 2018). Con este ejemplo podemos destacar lo siguiente: ¿Cómo se realiza un monitoreo de efectividad biológica de insecticidas en chinches si no existe una directriz en ninguno de los instrumentos legislativos?, sabiendo que la prevalencia de esta enfermedad es variable en todos los estados ¿Qué plaguicidas se deben aplicar en los estados más afectados? En lugares afectados por más de una ETV con diferentes grupos de vectores ¿Cuáles son las acciones establecidas en un instrumento normativo oficial para discriminar entre productos efectivos $\mathrm{y}$ no efectivos atendiendo a vectores de grupos taxonómicos diferentes (chinches y mosquitos, por ejemplo)? Es evidente que existen áreas de oportunidad para implementar un MIV transparente, preciso y eficaz.

Otro de los problemas relacionados al control químico por medio de plaguicidas es que su aplicación no es exclusiva del área de la salud, la aplicación de insecticidas también ocurre en la industria agrícola. Los insectos vectores pueden estar expuestos a los compuestos químicos de uso agrario $\mathrm{y}$, a su vez, durante una exposición prolongada, desembocar en una presión de selección que culminará en el desarrollo de resistencia. En la implementación del MIV la OMS sugiere un control sobre el uso de insecticidas agrícolas para evitar el fenómeno descrito anteriormente. En México no existe una base de datos, registro, o algún método efectivo que permita evaluar cuales insecticidas se utilizan en campos agrícolas, su frecuencia de uso, y por lo tanto se ignora si estos representan una amenaza para un manejo de vectores efectivo. Por lo tanto, es necesario la creación de un sistema de información que permita conocer por zonas, ciudades, o municipios, los plaguicidas utilizados en todos los ámbitos. Otra de las insuficiencias que hace falta solucionar dentro del territorio nacional es la aplicación de nuevos procedimientos para el estudio de resistencia a insecticidas. En México se llevan a cabo bioensayos de efectividad biológica (KuriMorales, et al. 2017), no obstante, estos pueden carecer de sensibilidad teniendo como consecuencia que la detección de resistencia ocurre cuando los mecanismos involucrados sean irreversibles. Para ello es ineludible establecer dentro de la normativa oficial mexicana estudios de monitoreo de resistencia que no solo involucren la aparición fenotípica, sino que permitan identificar los principales mecanismos metabólicos o genómicos que intervienen en la aparición de este fenómeno (Dusfour, et al. 2019). De manera similar, la recomendación de plaguicidas por parte del CENAPRECE requiere de un procedimiento estandarizado, documentado, transparente, normado y basado en evidencia científica que evite la aparición de contrariedades tales como las que se registran en la lista de productos recomendados para el combate de insectos vectores del 2020 (CENAPRECE, 2020). Para demostrar estas contrariedades es preciso relacionar esta lista a los resultados finales de adulticidas del año 2018 (CENAPRECE, 2018) donde se evaluó la susceptibilidad de Aedes aegypti a diferentes insecticidas utilizando el bioensayo en botella establecido por la CDC. De los 12 compuestos adulticidas probados, ocho de ellos (alfacipermetrina, clorpirifos, clotianidina, fenotrina, imidacloprid, propoxur, transcifenotrina y transflutrina) no tienen dosis diagnosticas establecidas en el manual del CDC lo que sugiere que las dosis usadas para estos químicos podrían no ser las óptimas. En cuanto a los resultados obtenidos, la fenotrina y el imidacloprid presentan un promedio de mortalidad de apenas $6 \%$ y $12 \%$, respectivamente. Mas a detalle, los resultados de mortalidad por estado muestran que solo para Yucatán en el caso de la fenotrina y Chihuahua para la Imidacloprid presentan una mortalidad superior al $98 \%$. A pesar de estos resultados ambos compuestos aparecen como insumos recomendados para su uso en 2020 y solo en el caso de la fenotrina se presenta una advertencia de uso condicionado. Tal advertencia carece de especificidad ya que no expresa explícitamente las acciones a tomar. Para esto la OPS (OPS, 2019) sugiere que la principal recomendación sea evaluar 
el uso de otro insecticida con diferente mecanismo de acción. Este tipo de ambigüedades podrían evitarse estableciendo en una norma oficial sobre la evaluación, elección y aplicación de insecticidas para el control de organismos vectores y con ello se fortalecen las políticas públicas de intervención de México.

La participación de centros de investigación o instituciones de educación superior es un elemento de apoyo que podría emplearse para la resolución de problemas en la aplicabilidad del MIV, mismo que es mencionado en el manual de MIV de la OMS. La NOM 032 indica que se debe promover los programas de vigilancia, prevención y control de las ETV en centros de investigación, instituciones de educación superior para un manejo adecuado de estas enfermedades. Al igual que el punto anterior, no existe un documento escrito que sirva como base para intensificar esta participación y como única línea de colaboración, los plaguicidas son evaluados por instituciones de educación superior para conocer su efectividad biológica.

Otras de las grandes disparidades entre el MIV recomendado por la OMS y la aplicación en México es la eficiencia en el uso de recursos y el ajuste de las medidas debido al cambio climático y la urbanización. La norma 032 muestra que cada una de las ETV sujetas a vigilancia en México cuenta con un MIV especifico y no se aprovecha el enfoque integral que promueve la OMS. Esto supone que se destinen recursos para distintas ETV en lugar de agrupar ciertas enfermedades con características similares. Por ejemplo, uno de los principales enfoques para el control de enfermedades transmitidas por mosquitos es el manejo de sitios de desarrollo larvario. Para evitar confusiones o extensiones innecesarias, convendría organizar un grupo de enfermedades como transmitidas por moquitos y establecer que uno de los principales medios de control sea el uso de vivienda y agua segura indicando todos los sitios de desarrollo identificados para todas las especies de mosquitos. Otra aproximación a la eficiencia en el uso de recursos y al mismo tiempo diversificar el uso de insecticidas para evitar el surgimiento de resistencia a un grupo químico en particular, se recomendaría la aplicación de insecticidas de diferentes grupos químicos a más de dos órdenes de insectos transmisores de enfermedades, claramente con la previa demostración de efectividad biológica.
Aunado a estas recomendaciones, se desconoce la manera en que los laboratorios nacionales investigan la biología de vectores, componente también mencionado en el MIV de la OMS. Ninguno de los instrumentos legislativos evidencia si existe investigación continua para determinar otras maneras de control aparte de las mencionadas en la NOM 032, mismas que solo incluyen manejo físico del hábitat, control químico, y control educativo a pesar de que se mencionan otro tipo de medidas.

Por último, vale la pena señalar que uno de los pilares del MIV en México incluye la comunicación social. Esto constituye un acierto en el MIV aplicado en la república ya que aquí se da la transmisión de la información a través de diferentes medios como la televisión, internet, folletos, o mensajes plasmados en la vía pública. No obstante, no existe un instrumento que permita determinar si la información dada a la población es tomada en cuenta por los habitantes de las zonas de riesgo. Realizar evaluaciones sobre la información que posee la población en general podría ayudar a intensificar los esfuerzos actuales o a corregir el rumbo de la transmisión de conocimiento.

Proyecto de respuesta mundial para el control de vectores 2017-2030

Para finalizar el análisis de los esfuerzos para el control de ETV en México se discute la respuesta mundial para el control de vectores 2017-2030 (OMS, 2020). Este proyecto se da para la mejora de ciertos componentes que permitirán un control de vectores eficaz, sostenible y adaptado que reduzca la amenaza de las ETV. La mayor parte de este documento incluye lo anteriormente propuesto por el manual de aplicación del MIV de la OMS. Además, se da en el marco de las principales ETV: el paludismo, dengue, filariasis linfática, enfermedad de Chagas, oncocercosis, leishmaniasis, fiebre del chikungunya, enfermedad por virus del Zika, fiebre amarilla, encefalitis japonesa y la esquistosomiasis. De estas, solo tres de ellas no poseen una vigilancia establecida en la NOM 017. Los objetivos de esta propuesta incluyen la reducción de la mortalidad en al menos en un $75 \%$, la reducción de la incidencia en al menos un $60 \%$ y la prevención de epidemias en países endémicos y no endémicos. Parte de su justificación destaca la falta de programas nacionales de investigación, participación efectiva multisectorial y comunitaria, además de débiles 
sistemas de monitoreo e implementación de nuevas intervenciones del control de vectores. Nuevamente, esta aproximación señala el constante cambio social, demográfico y ambiental que modifica las condiciones de transmisión propiciando la expansión geográfica de las ETV o la ampliación de periodos epidémicos.

La pieza fundamental de este nuevo enfoque envuelve la investigación básica y aplicada para el control de vectores en conjunto con un personal operativo altamente capacitado y metodologías innovadoras que remplacen o se apliquen simultáneamente a las todavía utilizadas. La investigación se propone al igual que hace tiempo, involucrar a las comunidades científicas nacionales e internacionales para la resolución del problema de las ETV. En cuanto a las nuevas propuestas se encuentra la capacitación del personal. Esta capacitación está orientada a la Entomología Médica para asegurar que las personas que operan a nivel local tengan los conocimientos suficientes sobre biología de vectores, epidemiología, métodos de control y monitoreo de resistencia. Reiteradamente, se anuncia la colaboración intersectorial para la mejor toma de decisiones y evitar la centralización de la información.

A escala regional la OPS (OPS, 2019) en su documento operativo del MIV para las Américas demarca brechas estructurales encontradas para la aplicación del manejo de vectores. Parte de estas brechas coinciden con lo descrito anteriormente en este texto y pone en evidencia la necesidad de nuevos enfoques. Por ejemplo, la OPS señala la falta de cobertura y calidad en las intervenciones en contra de la malaria, además de carencia de datos entomológicos, deficiencia en la estratificación del territorio para conocer áreas de riesgo prioritarias, capacitación de recursos humanos inadecuada, complicaciones con el proceso de compra de insecticidas y una ausencia de participación intersectorial que aplica tanto para la malaria como otras ETV. Aparte de estas deficiencias la OPS reconoce que existe una ineficacia en el control de las enfermedades ya que existe un tratamiento separado para cada enfermedad contrario a un enfoque integral. En cuanto a la normatividad, la OPS admite la importancia de los marcos normativos que deben implementarse en el nivel operativo más bajo.

\section{Conclusiones}

En México existe un esfuerzo para controlar el impacto de las ETV, la mayor parte de estos esfuerzos están establecidos en una serie de documentos oficiales como la LGS y las distintas normas oficiales mexicanas.

En comparación con la metodología propuesta por la OMS para el MIV y el proyecto más reciente para el control de vectores, la legislación nacional presenta discrepancias que, al adecuarse, podría incrementar la efectividad de las acciones en contra de los organismos vectores de manera sostenible, ecológica y socialmente aceptables.

Las políticas deben ser explícitas y claras para evitar ambigüedades y malas interpretaciones y deben planearse, ejecutarse y evaluarse con una colaboración interdisciplinaria, interinstitucional e interprofesional, de esta manera se lograrán establecer mejores condiciones para el manejo y eliminación de las ETV en nuestro país. Además, las políticas deberán fortalecer las iniciativas mundiales en materia de control de ETV.

\section{Bibliografía}

Beier, J. C. Keating, J. Githure, J. I. Macdonald, M. B. Impoinvil, D. E. Novak, R.J. (2008). Integrated vector management for malaria control. Malaria Journal; 7(S1).

Centro Nacional de Programas Preventivos y Control de Enfermedades. (2018). Resultados Finales Adulticidas 2018. Disponible en: https://www.gob.mx/cms luploads/attachment/file/545443/Resultados_Fin ales_Adulticidas_2018.pdf.

Centro Nacional de Programas Preventivos y Control de Enfermedades. (2020). Productos recomendados por el CENAPRECE para el combate de insectos vectores de enfermedades a partir de 2020. Disponible en: https://www.gob.mx/cms/uploads/attachment/fil e/546678/Productos_Recomendados_por_el_CE NAPRECE_para_el_Combate_de_los_Insectos_ Vectores_2020.pdf. 
Diario Oficial de la Federación. Reglamento de la Ley General de Salud en Materia de Sanidad Internacional. (1985). Recuperado de: http://www.diputados.gob.mx/LeyesBiblio/regle y/ Reg_LGS_MSI.pdf

Diario Oficial de la Federación. (2013). Norma Oficial Mexicana NOM-017-SSA2-2012. Para la vigilancia epidemiológica. Recuperado de: http://187.191.75.115/gobmx/ salud/documentos/manuales/00_NOM-017SSA2-2012_para_vig_epidemiologica.pdf

Diario Oficial de la Federación. (2015). Norma Oficial Mexicana NOM-032-SSA2-2014. Para la vigilancia epidemiológica, prevención y control de las enfermedades transmitidas por vector. Recuperado de: http://www.cenaprece.salud.gob.mx /programas/interior/vectores/descargas/pdf/NO M_032_SSA2_2014.pdf

Diario Oficial de la Federación. (2020). Constitución política de los Estados Unidos Mexicanos. Recuperado de: http://www.diputados.gob.mx/ LeyesBiblio/pdf/1_060320.pdf

Dusfour, I. Vontas, J. David, J.P. Weetman, D. Fonseca, D.M. Corbel, V. ... Chandre, F. (2019). Management of insecticide resistance in the major Aedes vectors of arboviruses: Advances and challenges. PLOS Neglected Tropical Diseases 13(10):e0007615. doi: 10.1371/journal.pntd.0007615.

Gallup, J. Sachs, J. (2001). The economic burden of malaria. The American Journal of Tropical Medicine and Hygiene 64(1_suppl):85-96.

González, L. Hernández, D. (2017). Malaria: Vectores. Ciencia 68(1):50-53.

Kuri, P. A. Correa, F. González, C. Moreno, M. Santos, R. Román, S. ... González, J. F. (2018). Insecticide susceptibility status in Mexican populations of Stegomyia aegypti (=Aedes aegypti): a nationwide assessment. Medical and Veterinary Entomology 32(2):162-174. doi: $10.1111 /$ mve. 12281 .
Ley General de Salud. (2020). Recuperado de: http://www.diputados.gob.mx/ LeyesBiblio/pdf/142_240120.pdf

Mullen, G.R. Durde, L.A. (2009). Medical and veterinary entomology. Amsterdam, Países bajos: Elsevier.

Organización Mundial de la Salud (OMS). (2020). Enfermedades transmitidas por vectores. Recuperado de: https://www.who.int/es/newsroom/fact-sheets/detail/vector-borne-diseases

Organización Mundial de la Salud. (2008). Reglamento Sanitario Internacional. 2a edición. Ginebra.

Organización Mundial de la Salud. (2012). Handbook for integrated vector management. Biological and Environmental Control of Disease Vectors. Ginebra.

Organización Mundial de la Salud. (2019). World Malaria Report 2019. Ginebra.

Organización Mundial de la Salud. (2020). Respuesta mundial para el control de vectoresenfoque integrado para el control de las enfermedades de transmisión vectorial, 2020 Disponible

en: http://www.who.int/malaria/areas/vector_control /Draft-WHO-GVCR-2017-2030-esp.pdf

Organización Panamericana de la Salud. Documento operativo de aplicación del manejo integrado de vectores adaptado al contexto de las Américas, 2019. 55.

Patterson, J. Sammon, M. Garg, M. (2016). Dengue, Zika and Chikungunya: Emerging Arboviruses in the New World. Western Journal of Emergency Medicine 17(6):671-679. doi: 10.5811/westjem.2016.9.30904.

Pita, S. Mora, P. Vela, J. Palomeque, T. Sánchez, A. Panzera, F. ... Lorite, P. (2018). Comparative Analysis of Repetitive DNA between the Main Vectors of Chagas Disease: Triatoma infestans and Rhodnius prolixus. International Journal of Molecular Sciences 19(5):1277. doi: 10.3390/ijms19051277. 
Rojo-Medina, J. Ruiz-Matus, C. Salazar-Schettino, PM. González-Roldán, JF. (2018). Enfermedad de Chagas en México. Gaceta de México;154(5).

Schwartz, E. (2012). Prophylaxis of Malaria. Mediterranean Journal of Hematology and Infectious Diseases 2012045. doi: 10.4084/mjhid.2012.045.

Shepard, D.S. Undurraga, E.A. Halasa, Y.A. Stanaway, J.D. (2016). The global economic burden of dengue: a systematic analysis. The Lancet Infectious Diseases 16(8):935-941. doi: 10.1016/S1473-3099(16)00146-8.

Sridhar, S. Luedtke, A. Langevin, E. Zhu, M. Bonaparte, M. Machabert, T. ... Diaz, C. A. (2018). Effect of Dengue Serostatus on Dengue Vaccine Safety and Efficacy. New England Journal of Medicine 379(4):327-340. doi: 10.1056/NEJMoa1800820.

Velásquez Gavilanes, Raúl (2009). Hacia una nueva definición del concepto "política pública". Desafíos, 20,149-187. [fecha de Consulta 10 de febrero de 2021]. ISSN: 0124-4035. Disponible en:

https://www.redalyc.org/articulo.oa?id=3596/35 9633165006 